Review

\title{
AD biomarker discovery in CSF and in alternative matrices
}

\author{
Giulia Sancesario $^{\mathrm{a}, *}$, Sergio Bernardini ${ }^{\mathrm{b}}$ \\ ${ }^{a}$ Dept. Clinical and Behavioural Neurology, Santa Lucia Foundation, Rome, Italy \\ ${ }^{\mathrm{b}}$ Dept. Experimental Medicine, University of Tor Vergata, Rome, Italy
}

\section{A B S T R A C T}

Core Alzheimer's Disease (AD) biomarkers are the cerebrospinal fluid (CSF) proteins amyloid $\beta 42$ and $\beta 40$, and the tau proteins, total and phosphorylated. Their use is recommended by research guidelines for diagnostic purpose and for stratification of patients for clinical trials. However, novel potential biomarkers are needed, which can mirror risk factors and other different mechanisms of the disease, hopefully in less invasive biological fluids or matrices. Studies on blood, urine, saliva, tears gave promising results, and several novel molecules have been identified, as potential brain derived biomarkers, thanks to the development of novel ultrasensitive technologies. In this review, we discuss about advantages and limits of the classical CSF biomarkers of AD, as well as of novel CSF candidate biomarkers and recent promises from alternative matrices.

\section{Introduction}

The development of an effective therapy for Alzheimer's Disease (AD) is yet like the "quest for the Grail", something that you search but that is very hard to get. To pursue this goal, the discovery of novel biomarkers may improve diagnostic sensitivity and help to achieve more precise therapeutic targets. The "precision medicine" indeed focuses on identifying which approaches will be effective for which patients, based on the individual assessment of biological, genetic and other risk factors.

Currently, the core AD biomarkers are the cerebrospinal fluid (CSF) proteins amyloid $\beta 42$ and $\beta 40$, and the tau proteins, total and phosphorylated, directly related to the specific AD pathology. Their use is recommended by research guidelines for diagnostic purpose and for stratification of patients for clinical trials. However, novel potential biomarkers are needed, which can mirror also risk factors and other different mechanisms of the course of the disease, hopefully in less invasive biological fluids or matrices. So far, hundreds of candidate biomarkers have been investigated, but their involvement in the AD pathophysiology and their usefulness in clinical practice have to be validated yet, assessing their sensitivity and specificity [1]. Actually, in clinical practice exceptionally a test has the ideal $100 \%$ sensitivity and specificity, giving the certainty of the diagnosis; in reality, just tests with sensitivity and specificity values above $90 \%$ are considered to have credibility, giving high probability, but not certainty in differential diagnosis. In this review, we discuss about the clinical relevance of classical CSF biomarkers of $\mathrm{AD}$, as well as of novel emerging CSF candidate biomarkers and recent promises from alternative non-invasive matrices.

\section{Cerebrospinal fluid is the main source for neurodegenerative biomarkers}

For neurodegenerative diseases, such as $\mathrm{AD}, \mathrm{CSF}$ is the main source for investigating substances released by brain tissue and eventually representing biomarkers of disease, because it is draining the interstitial fluid in near proximity with the cells of the central nervous system and can reflect modifications occurring during the disease [2]. In AD pathology, biomarkers can represent the biological evidence of the pathological processes measurable in vivo, and their measurement is a useful tool for improving the accuracy as well as for allowing an early and timely diagnosis. Aside AD, the CSF represents the main source for the detection of the protein 14-3-3, considered in the past the Gold Standard for the in vivo diagnosis of Creutzfeldt-Jakob Disease (CJD) included in the diagnostic criteria by the World Health Organization (WHO) [3]. However, in recent years the 14-3-3 assay, although of moderately high diagnostic accuracy, has been considered an imperfect test by the Evidence-based Guideline of the American Academy of Neurology (2012), since the test lacks the diagnostic accuracy either to include a CJD diagnosis as a possibility or to rule out CJD. Recently, a novel ultra-sensitive assay, the Real-Time Quaking-Induced Conversion (RT-QuIC) has been developed, exploiting the seeding ability of the pathogenic Prion Protein (PrP) in the CSF [4], and its use has been accepted for the premortem diagnosis of CJD [5], with the $92 \%$ sensitivity and the $100 \%$ specificity [6].

\section{1. $C S F$ core $A D$ biomarkers}

In $\mathrm{AD}$, modification of the CSF levels of amyloid $\beta 42(\mathrm{~A} \beta 42)$, total

\footnotetext{
* Corresponding author at: Dept Clinical and Behavioural Neurology, Santa Lucia Foundation, via Ardeatina 354, Rome, Italy.

E-mail address: g.sancesario@hsantalucia.it (G. Sancesario).
} 
and phosphorylated tau (T-tau and p-tau), mirror the tissue formation of amyloid plaques and neurofibrillary tangle, respectively, the neuropathological hallmarks of the disease $[7,8]$. The decrease of CSF A 342 , and more recently and accurately, the decrease of the amyloid ratio $\mathrm{A} \beta 42 / \mathrm{A} \beta 40$, together with the increase of both T-tau and p-tau constitute the so called "CSF AD profile", which can be useful for establishing a correct diagnosis especially in case of ambiguous clinical diagnoses of dementia [9]. Moreover, the assessment of CSF biomarkers has a predictive value in asymptomatic or prodromal phase of the disease, and helps to identify those patients with mild cognitive impairment (MCI) that are stable or will develop other forms of dementia [10-12]. Furthermore, CSF biomarkers can identify AD patients with rapidly progressive dementia [13,14], and can be associated with clinical phenotype, specific functional traits or cholinergic dysfunction $[15,16]$. Recently, a systematic review and meta-analysis evaluated the data from literature for classical and emerging biomarkers of AD, in CSF and blood, on a total of 15,699 patients with $\mathrm{AD}$ and 13,018 controls. The study confirms the strong association of the core CSF biomarkers with $\mathrm{AD}$ and $\mathrm{MCI}$ due to $\mathrm{AD}$, encouraging their use in both clinical practice and research [17].

In 2011, CSF biomarkers have been included in the recommendations for the diagnosis of pre-clinical, MCI, and $\mathrm{AD}$ dementia by both the National Institute on Aging and Alzheimer's Association (NIA-AA) $[18,19]$ and the International Working Group (IWG), with a revision updated in 2014 [20]. In 2018, NIA-AA recommendations were further updated, unifying imaging and biological markers in a research framework, and grouping biomarkers in three categories or AT(N) groups, indicating amyloid deposition (A), pathologic fibrillary tau (T), and neurodegeneration (N). Group A includes low CSF Ab42 or cortical amyloid PET ligand binding; group $\mathrm{T}$ includes elevated CSF phosphorylated tau (P-tau) and cortical tau PET ligand binding; group "(N)" includes elevated CSF T-tau, FDG PET hypometabolism, and atrophy on MRI. The characteristics of group A and $\mathrm{T}$ define the specific $\mathrm{AD}$ abnormal protein deposits as a unique neurodegenerative disease among different disorders that can lead to dementia. The characteristics of group " $(\mathrm{N})$ " define biomarkers of neurodegeneration or neuronal injury which also occur in non-AD conditions, particularly in elderly individuals where comorbidities are common, separating tau pathology specific for $\mathrm{AD}$, from tau and other biomarkers that are nonspecific measures of neurodegeneration/neuronal injury. New emerging biomarkers can be incorporated within the existing AT(N) groups, such as neurofilament light chain both in blood or in the CSF, or CSF neurogranin, to be included in the "N" category. The AT(N) classification provides a research framework for grading disease severity using biomarkers: categories in group A and T are measures used to define AD and must be specific for the disease, whereas neurodegenerative/ neuronal injury biomarkers in group $(\mathrm{N})$ are non-specific for $\mathrm{AD}$, and are used only to stage severity not to define the presence of the Alzheimer's continuum. However, none of the biomarkers in the AT(N) groups are as sensitive as direct examination of tissue at autopsy. Indeed, there is not correspondence between the disease severity graded on biomarkers' profiles, and the different cognitive stages, because we cannot know if the cognitive deficit is attributable to $\mathrm{AD}$ alone or to other potential comorbidities in addition. Accordingly, different algorithms have been developed based on the biomarker profile and cognitive stage to assess risk of short-term cognitive decline and to define the descriptive nomenclature of the syndromal cognitive staging, alternative to traditional syndromal categories, dividing the cognitive continuum into three stage - Cognitively Unimpaired, MCI, and dementia, with dementia further subdivided into mild, moderate, and severe stages. However, these three syndromal categories, have been codified for clinical practice in the Diagnostic and Statistical Manual of Mental Disorders, Fifth Edition, criteria by the terms "mild neurocognitive disorder" (essentially MCI) and "major neurocognitive disorder" (essentially dementia) [21].

\subsection{Novel CSF candidate biomarkers}

The research of novel fluid markers may have great advantages as tool for the diagnosis and clinical progression of $\mathrm{AD}$ and other neurodegenerative diseases. In fact, compared to imaging or neuropsychological examination, the cost of CSF biomarkers is relatively less expensive, and prices are expected to decrease dramatically with new random-access platforms opening commercial concurrence. A number of candidate molecules including Beta secretase1 (BACE1), Triggering receptor expressed on myeloid cells 2 (TREM2), neurogranin, $\alpha$-synuclein, neurofilament (NF), and many others, have been investigated as potential biomarkers of $\mathrm{AD}$. These proteins are associated with pathological mechanisms or to other aspects of $\mathrm{AD}$ pathophysiology, such as biochemical modifications, enzymatic deficits, clearance and degrading pathways [22,23]. For example, the neurofilaments are axonal protein which can be of high (NF-H), intermediate (NF-I) and light (NF-L) molecular weight. NFs are released in the extracellular space and are a marker for neuronal damage and degeneration. In the CSF, level of NF-L increases as result of diffuse neuronal damage in many neurodegenerative diseases, such as Multiple Sclerosis, Frontotemporal Dementia and $\mathrm{AD}[17,24,25]$.

However, the values of such molecules as biomarkers for $\mathrm{AD}$ or other dementias have yet to be validated [26]. Moreover, a widespread diffusion of CSF biomarkers in the integrated diagnosis for AD in clinical practice is hampered by different factors, e.g. the fear regarding the lumbar puncture, economic considerations, or the skepticism regarding the value of biomarkers in clinical practice $[27,28]$. So, there is a need to identify additional reliable, reproducible, non-invasive, simple to measure, and inexpensive fluid biomarkers in alternative matrices, e.g. blood, urine, saliva and other (Fig. 1). The development of a reliable blood-based or alternative matrix biomarkers easily implementable into large populations may be of paramount help.

\section{Biomarkers discovery in alternative matrices}

\subsection{Biomarkers in blood}

The blood is separated from the CSF and the central nervous system (CNS) by the blood-brain-barrier, but a selected low amount of brainderived proteins can cross the barrier reaching the bloodstream, and vice versa. Therefore, we could search in the blood specific substances deriving by exclusive brain pathologies, or substances deriving by systemic disease processes involving also the brain. Oppositely to CSF, blood is a more complex matrix for investigating neuronal processes, for different reasons. In fact, blood contains a high number of cells and molecules, such as different classes of proteins, immunoglobulins, hormones, cytokines, lipids, etc. rendering the investigation into this matrix challenging, due to the potential analytical interference with the traditional immunoassay platforms. Then, the activity of proteases or protein carriers can participate in degrading or masking the epitopes of

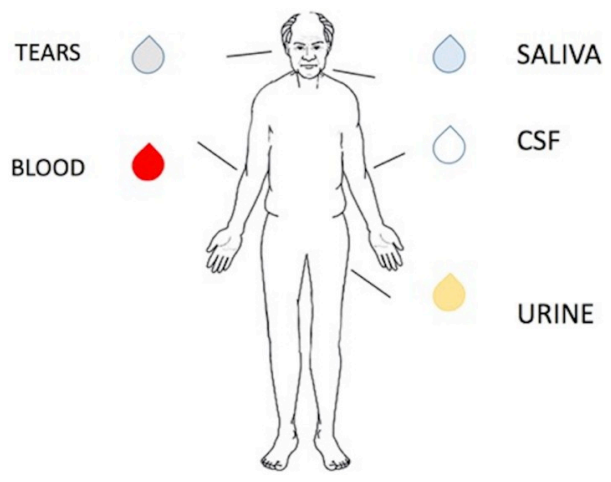

Fig. 1. Alternative matrices for biomarkers discovery. 
pathological markers, which finally interfere with their detection and measurement. Moreover, the low amount for brain derived biomarkers in blood require highly sensitive technologies for the detection, while the investigation of blood derived proteins implicates a systemic origin of the disease and a secondary probable involvement of the CNS. For all these and many other reasons, the research of neurodegenerative biomarkers in alternative fluids is challenging and, to date, the use of blood biomarkers for the diagnosis of $\mathrm{AD}$ has not been validated.

Several studies on plasma biomarkers have indeed shown conflicting results, even for the core $\mathrm{AD}$ biomarkers. The measurement of amyloid entities in blood would be of great interest, but it would be also difficult to have clear interpretation. In fact, although it is well known that $A \beta$ peptide is mainly produced by brain cells, astrocytes and neurons $[29,30]$, an alternative hypothesis thus supposed that $A \beta$ peptide can spread to the brain from the blood, similarly to other amyloidosis which are characterized by the abnormal extracellular deposition of misfolded amyloid proteins. In blood, platelets are the primary source of $A \beta$ peptide producing about $90 \%$ of total blood $A \beta$ [21]. This platelets' secreted $A \beta$ is similar to that found in the senile plaques of AD patients, and is increased in vivo in patients with familiar form of $\mathrm{AD}$, which are due to the mutations of presenilin-1 and -2 or Amyloid Precursor Protein (APP) [31]. Moreover, proteins may be regulated and modified by different processes in the CNS versus the periphery, resulting in a lack of correlation between abundance in CSF and blood. A possible imbalance between the $\mathrm{A} \beta$ production/degradation process may contribute to the pathogenesis of $\mathrm{AD}$, and different proteolytic systems have been demonstrated to regulate $A \beta$ peptide levels in $\mathrm{AD}[22,32]$.

Oppositely to the significant decrease of $A \beta 42$ and $A \beta 42 / A \beta 40$ ratio in $\mathrm{CSF}$ of $\mathrm{AD}$ patients, their level in blood have been found to be unaltered or having only small changes in patients respect to control [17]. This finding, together with the evidence that the level of plasma A 342 does not correlate with the CSF concentration, leads to hypothesize a different metabolism for amyloid- $\beta$ in peripheral tissues [33].

In order to overcome the limit in the detection encountered using traditional ELISA platforms, novel approaches and technologies are emerging with the potential to provide superior sensitivity and specificity for measuring $A \beta$, tau and novel candidate biomarkers in blood samples. Among others, the most promising technologies appear to be the mass-spectrometry analysis (MS) coupled with immunoprecipitation, new immunoaffinity-based assays, including immunomagnetic reduction (IMR), electrochemiluminescence, and the single molecule array (SIMOA) [34]. In ImmunoMagnetic Reduction (IMR), magnetic nanoparticles are coated with specific monoclonal antibodies, and detected via magnetic interaction. Briefly, magnetic nanoparticles are incubated with biological samples, binding with the antigens to be detected; due to the association with the antigen, magnetic nanoparticles become larger, with a reduction of the oscillation amplitude under the magnetic field, which corresponds to the amount of bound antigen [35]. A preliminary study using SQUID-based IMR assayed plasma amyloid and total Tau protein in a small group of patients ( $n=39$ ) with subjective cognitive decline or mild cognitive impairment. The results showed a diagnostic accuracy for prodromal AD higher than $80 \%$ and reveal the possibility of screening for early-stage AD [36].

MS techniques have the advantage of allowing the investigation of different species of $A \beta$ peptides at very low concentration. Immunoprecipitation is used to pull down various $A \beta$ fragments in plasma samples followed by MS analysis, reaching a sensitivity of 2.5-40 pM for A $\beta 42$ and 10-160 pM for A $\beta 40$ [37]. The sensibility of MS may indeed be enhanced by combining different sources, analyzers and detectors and by the use of MS in tandem with chromatographic or other separation techniques [38]. Panels of novel candidate biomarkers have been identified in plasma and CSF of AD patients as well as in cognitively unimpaired subjects. Such putative biomarkers or predictive panel are related to $A \beta$-peptide processing pathway, or to different metabolic pathways, mostly not directly related to $A \beta$ pathology per se, such as the serine protease prothrombin (a precursor to thrombin), NGN2, FHAD1, and DNAH10, and many others [39].

Several MS studies have shown that differences in the plasma level of $A \beta 42, A \beta 42 / 40$ ratio or APP species can identify A $\beta$-PET positive individuals with high sensitivity and specificity in both MCI and $\mathrm{AD}$ patients, as well as in subjects without cognitive impairment $[37,40]$.

SIMOA is a digital form of ELISA, which uses individual immunocomplexes on paramagnetic beads, in thousands of femtoliter sized wells, reaching a very high sensitivity $(0.019 \mathrm{pg} / \mathrm{ml}$ for tau and $0.044 \mathrm{pg} / \mathrm{ml}$ for A 342 ). Using SIMOA technique, ultrasensitive assays for the measurement of brain derived proteins in plasma have been developed, including amyloid, tau and NF-L. Different studies found positive correlations between the level of plasma and CSF A 342 and A $\beta 42 / 40$ ratio [41,42], and a negative correlation with brain amyloid deposition [41]. In subject with MCI or subjective cognitive impairment, and preclinical or prodromal $\mathrm{AD}$, the level of plasma $\mathrm{A} \beta 42$ was slightly decreased [41].

The plasma tau protein has been broadly investigated in AD, with the attempt to detect peripheric alterations associated with the neuronal damage occurring in the brain. Results from studies using classical enzyme-linked immunoassays shown controversial results, and have the limit of small population of patients enrolled. In different studies, levels of plasma tau were found to be highly increased [43-45], moderately increased [46], unaltered [47] or decreased [48,49] in AD group.

A recent study evaluated the correlations between plasma tau and clinical data, CSF and imaging markers, by using an ultrasensitive method (with the Human Total Tau kit (research use only grade, Quanterix, Lexington, MA), on the Simoa HD-1 analyzer, in a large number of patients from the Biomarkers for Identifying Neurodegenerative Disorders Early and Reliably (BioFINDER) study and the Alzheimer's Disease Neuroimaging Initiative (ADNI). Authors found higher level of plasma tau in patients with $\mathrm{AD}$ dementia, but in part overlapped with normal aging and in patients without dementia; interestingly, in this study a prognostic value for plasma tau concentration has been observed, allowing to identify cognitive impairment or brain atrophy and hypometabolism during the follow-up [50]. Notwithstanding, despite the differences observed among groups, these findings do not support the use of plasma tau as an $\mathrm{AD}$ biomarker in individual people.

Moreover, in a review and meta-analysis study, Olsson et al. found that, oppositely to CSF, data from $22 \mathrm{AD}$ and 20 control cohorts on a total of 15,699 patients with $\mathrm{AD}$ and 13,018 controls showed that the level of $A \beta 42$ and $A \beta 40$ in blood were not statistically different; whereas, only a significant difference was confirmed for the increase of plasma t-tau in $\mathrm{AD}$ compared to controls [17].

Recently, a study using IMR found a correlation between plasma ptau181 level and AD severity, with level of plasma p-tau181 significantly higher in mild $\mathrm{AD}$ respect to $\mathrm{MCI}$ due to Ad and to healthy subjects [51].

A great variability of the results in relation to the methodology used has been observed and represents a limitation for the use of these markers. Several studies that have evaluated biological markers in the blood have shown conflicting results [52]. For example, increased A $\beta 42$ was reported in $\mathrm{AD}$ patients compared to controls by using IMR assay $[43,44]$; other studies on the same biomarker measured with SIMOA found significant decreased concentration in $\mathrm{AD}$ respect to $\mathrm{MCI}$ and control subjects [41]. On the other hand, significant increase of plasma tau level was observed in AD compared to control, by using both SIMOA or IMR platforms.

Interestingly, the plasma level of NF-L, a marker of axonal damage, is correlated with the concentration in CSF, representing a useful screening test for neurodegenerative processes, or a potential monitoring biomarker of disease progression or therapy [42,53,54]. Level of NF-L were found to be highly increased in MCI- AD patients, and even 
more in severe $\mathrm{AD}$ respect to nondemented group, and moderately correlated with cognitive impairment evaluated by the Mini Mental State Examination across all patients [53]. In AD, the concentration is increased, and moreover, a potential predictive value has been found in a study on familial cases of $\mathrm{AD}$, were increased blood NF-L was observed in symptomatic as well as in presymptomatic carriers of $\mathrm{AD}$ mutations [55]; further, NF-L are predictive of cognitive decline [55].

Finally, a number of other emerging biomarkers or multi-marker panel have been investigated in different studies, aiming to find a potential link with the neurodegeneration, glial activation, $\mathrm{A} \beta$ pathology, systemic and inflammatory response to the disease, impaired protein [17] or degrading enzymes [56]. However, despite most of these candidate markers show significative association with the disease in CSF concentrations, the corresponding levels in blood do not reflect such modification [17].

\subsection{Saliva and tears}

Due to its complete lack of invasiveness, saliva represents an attractive matrix for the biomarkers' research. Moreover, saliva can be self-collected with no stress for the patients, and the collection can be repeated as necessary, thus rendering saliva an ideal marker for monitoring diseases. Most blood proteins used for diagnosis of different diseases can be found in saliva [57]. Moreover, several studies have demonstrated a versatile use of saliva for omics profiling [38,58,59]. Since salivary secretion is reduced with aging, it is expected that changes in the biochemical composition may be associated with the development of age-associated disorders.

Several methods have been developed for the measurement of $A \beta$ in saliva, by using ELISA, magnetically assembled antibody conjugated nanoparticles, Luminex assay or mass spectrometry following immunoprecipitation. Salivary A $\beta$ levels have been investigated by different studies but results are conflicting: $A \beta 42$ levels were found to be increased [60-62] or unaltered [63,64] in AD and MCI patients respect to controls or PD. Moreover, the level of salivary tau seems to not have a clinical usefulness, whereas the ratio $\mathrm{p}$-tau/tau is slightly increased in $\mathrm{AD}$ patients $[64,65]$.

Moreover, several candidate biomarkers have been investigated in saliva, including mucins, acetylcholinesterase, markers of oxidative stress, cortisol, etc. Unlucky, results should still be replicated in larger multicenter and longitudinal studies (for a review see [57].

Recently, a paper hypothesized the use of tears as tool for non-invasive translational studies in AD patients [66]. Authors investigated the modification in tears chemical composition in $23 \mathrm{AD}$ patients and control donors, by using quantitative proteomics approach. Interestingly, authors found modifications in the tear flow rate, total protein concentration and composition of the chemical barrier specific to AD. In particular, the combination of a panel of proteins, including lipocalin-1, dermcidin, lysozyme-C and lacritin had a good accuracy (81\% sensitivity and $77 \%$ specificity) in discrimination $\mathrm{AD}$ patients [66] supporting the use of tears for research purpose.

\subsection{Biomarkers in olfactory mucosa}

Olfactory mucosa has an extracranial location, and constitute a source of true neural progenitor cells. Although biopsies of the olfactory neuroepithelium (ON) are an established even though experimental procedure, successful in vivo collection of $\mathrm{ON}$ is possible by gentle non damaging brushing with wet cotton swab in the medial and superior side of the inferior nasal meatus. Olfactory cells become attached to the brush and can be studied. After nasal brushings, RT-QuIC analysis of PrP in the olfactory neuroepithelium has been shown to have a comparable specificity and improved sensitivity when compared with CSF analysis $[67,68]$.

\subsection{Biomarkers in urine}

Urine has so far represented an interesting source of markers, mainly for the diagnosis and monitoring of renal dysfunction, prostate and urinary tract cancers. Urine has the advantage of an easy and noninvasive collection, and the possibility of repeatable collections. Several cells, proteins, free DNA and microRNA, as well as extracellular vesicles, can be detected, which can reflect different pathological conditions.

Recently, growing evidences support the use of urine in a number of diseases, including AD. Dementia and kidney diseases seems to share common risk factors, as diabetes, high blood pressure and high cholesterol [69]. A review and meta-analysis shown that renal dysfunction is associated with increased risk of developing cognitive impairment or dementia [69]. Moreover, a meta-analysis including 5 studies, for a total of more than 27,000 persons, has demonstrated that albuminuria is associated with an increased risk of cognitive impairment or dementia of 35\%, although it has to be defined whether the association with albuminuria is causal or due to a common mechanism. Oppositely, no association was found for estimated glomerular filtration rate (eGFR) [70].

A recent study combined computational and experimental methods to identify potential urine biomarkers associated with $\mathrm{AD}$. Authors identify 559 genes encoding for urine-excretory proteins which were differentially expressed in AD brains. Among them, three proteins, SPP1 (Secreted Phosphoprotein 1), GSN (Gelsolin) and IGFBP7 (Insulin-Like Growth Factor-Binding Protein-7) were found to be differentially expressed in the urine of $\mathrm{AD}$ patients [71].

Several studies support the use of urinary Alzheimer-associated neuronal thread protein (AD7c-NTP) as potential candidate for the early diagnosis of AD. AD7c-NTP is a transmembrane phosphoprotein, which co-localizes with neurofibrillary tau tangle [72]. AD7c-NTP is associated with neuron accumulation of p-tau and its CSF increase in AD patients [73,74]. Concentration of urinary AD7c-NTP can predict $\mathrm{A} \beta$ brain deposition in $\mathrm{AD}$ and MCI patients with high accuracy [75]. Moreover, increased AD7c-NTP was observed in CSF and urine in the early phase of $\mathrm{AD}$ pathology, with positive association with the $\mathrm{AD}$ severity [76]. These data were confirmed by a meta-analysis, encouraging the use of urinary AD7c-NTP for the early diagnosis of probable AD [77].

Although promising, larger and longitudinal studies are needed in order to confirm the employment of urine $\mathrm{AD}$ biomarkers.

\section{Discussion}

One of the reasons for the failure of clinical trials for $\mathrm{AD}$ is the lack of an accurate panel of biomarker for establishing a correct diagnosis especially in case of ambiguous clinical dementia. The discovery of novel biomarkers for AD is a great challenge, and is strictly linked to the development of effective disease modifying therapies. Recent advances in molecular medicine, has leads to a novel concept named "precision medicine", which is characterized by the choose of the most effective therapy for each patient, based on an accurate stratification of patient profile. Any novel marker which can be helpful for this purpose is of paramount interest, in order to reach the classification of the population based on molecular, biological markers and environment factors.

The recent advance of novel ultrasensitive technologies has renewed the enthusiasm for the research of biomarkers in alternative body fluids, due to increasing accuracy and sensitivity of the detection. Further, the identification of biomarkers in non-invasive matrix is auspicable but not yet consolidated, to allow the screening of people at risk, to identify initial biochemical modifications in mild patients, and to monitor evolution of the disease.

Actually, there is a great expectation towards the discovery of new biomarkers in alternative matrices that can provide in vivo measurements of a series of further pathophysiological processes associated 
with the pathogenesis of AD. New molecules, including synaptic proteins and oligomers, will expand the range of markers covering the full spectrum of molecular events in the AD [78]. The use of panels in multi centers longitudinal clinical studies on large populations will provide essential additional information on the understanding of pathogenetic processes and on the development of effective disease modify therapies for $\mathrm{AD}$. However, the work is just in progress and not definite new biomarkers of neurodegenerations have been achieved.

\section{Concluding remarks}

In the future, the potential use of alternative matrices for the diagnosis and research of $\mathrm{AD}$ and other pathologies seems to be really interesting, notably due to their low invasiveness. The impact of plasma biomarkers on daily clinical practice could be substantial. Notwithstanding, some difficulties should be taken into account. The use of novel matrices has some limitations, for example the standardization of the preanalytical phase or technical problems linked to the small volume of samples. This should encourage rather than discourage the research.

\section{Declaration of Competing Interest}

None.

\section{References}

[1] D.G. Altman, J.M. Bland, Diagnostic tests. 1: sensitivity and specificity, BMJ 308 (1994) 1552.

[2] G.M. Sancesario, S. Bernardini, How many biomarkers to discriminate neurodegenerative dementia? Crit. Rev. Clin. Lab. Sci. 52 (2015) 314-326.

[3] World Health Organization, Manual for Strengthening Diagnosis and Surveillance of Creutzfeldt-Jakob Disease, WHO, Geneva, Switzerland, 1998.

[4] R. Atarashi, K. Satoh, K. Sano, T. Fuse, N. Yamaguchi, D. Ishibashi, et al., Ultrasensitive human prion detection in cerebrospinal fluid by real-time quakinginduced conversion, Nat. Med. 17 (2011) 175-178.

[5] D. Criteria, CDC's Diagnostic Criteria for Creutzfeldt-Jakob Disease (CJD), N.C.R.S.U, 2018

[6] A.J.E. Green, RT-QuIC: a new test for sporadic CJD, Pract. Neurol. 19 (2019) 49-55.

[7] A.M. Fagan, M.A. Mintun, R.H. Mach, S.Y. Lee, C.S. Dence, A.R. Shah, et al., Inverse relation between in vivo amyloid imaging load and cerebrospinal fluid Abeta42 in humans, Ann. Neurol. 59 (2006) 512-519.

[8] S. Palmqvist, H. Zetterberg, K. Blennow, S. Vestberg, U. Andreasson, D.J. Brooks, et al., Accuracy of brain amyloid detection in clinical practice using cerebrospinal fluid beta-amyloid 42: a cross-validation study against amyloid positron emission tomography, JAMA Neurol. 71 (2014) 1282-1289.

[9] E. Niemantsverdriet, B.F.E. Feyen, N. Le Bastard, J.J. Martin, J. Goeman, P.P. De Deyn, et al., Added diagnostic value of cerebrospinal fluid biomarkers for differential dementia diagnosis in an autopsy-confirmed cohort, J. Alzheimers Dis. 63 (2018) 373-381.

[10] O. Hansson, H. Zetterberg, P. Buchhave, E. Londos, K. Blennow, L. Minthon, Association between CSF biomarkers and incipient Alzheimer's disease in patients with mild cognitive impairment: a follow-up study, Lancet Neurol. 5 (2006) 228-234.

[11] N. Andreasen, L. Minthon, E. Vanmechelen, H. Vanderstichele, P. Davidsson, B. Winblad, et al., Cerebrospinal fluid tau and Abeta 42 as predictors of development of Alzheimer's disease in patients with mild cognitive impairment, Neurosci. Lett. 273 (1999) 5-8.

[12] P.J. Visser, F. Verhey, D. L. Knol, P. Scheltens, L. O. Wahlund, Y. Freund-Levi, et al, Prevalence and prognostic value of CSF markers of Alzheimer's disease pathology in patients with subjective cognitive impairment or mild cognitive impairment in the DESCRIPA study: a prospective cohort study, Lancet Neurol. 8 (2009) 619-627.

[13] G. Koch, L. Belli, T.L. Giudice, F.D. Lorenzo, G.M. Sancesario, R. Sorge, et al., Frailty among Alzheimer's disease patients, CNS Neurol. Disord. Drug Targets 12 (2013) 507-511.

[14] O. Hansson, J. Seibyl, E. Stomrud, H. Zetterberg J. Q Trojanowski, T. Bittner, et al, CSF biomarkers of Alzheimer's disease concord with amyloid-beta PET and predict clinical progression: a study of fully automated immunoassays in BioFINDER and ADNI cohorts, Alzheimers Dement. 14 (2018) 1470-1481.

[15] A. Martorana, Z. Esposito, F. Di Lorenzo, V. Giacobbe, G.M. Sancesario, G. Bucchi, et al., Cerebrospinal fluid levels of Abeta42 relationship with cholinergic cortical activity in Alzheimer's disease patients, J. Neural Transm. (Vienna) 119 (2012) $771-778$.

[16] T. Schirinzi, F. Di Lorenzo, G.M. Sancesario, G. Di Lazzaro, V. Ponzo, A. Pisani, et al., Amyloid-mediated cholinergic dysfunction in motor impairment related to Alzheimer's disease, J. Alzheimers Dis. 64 (2018) 525-532.

[17] B. Olsson, R. Lautner, U. Andreasson, A. Ohrfelt, E. Portelius, M. Bjerke, et al., CSF and blood biomarkers for the diagnosis of Alzheimer's disease: a systematic review and meta-analysis, Lancet Neurol. 15 (2016) 673-684.

[18] G.M. McKhann, D.S. Knopman, H. Chertkow, B.T. Hyman, C.R. Jack Jr. C.H. Kawas, et al., The diagnosis of dementia due to Alzheimer's disease: recommendations from the National Institute on Aging-Alzheimer's Association workgroups on diagnostic guidelines for Alzheimer's disease, Alzheimers Dement. 7 (2011) 263-269.

[19] M.S. Albert, S.T. DeKosky, D. Dickson, B. Dubois, H.H. Feldman, N.C. Fox, et al., The diagnosis of mild cognitive impairment due to Alzheimer's disease: recommendations from the National Institute on Aging-Alzheimer's Association workgroups on diagnostic guidelines for Alzheimer's disease, Alzheimers Dement. 7 (2011) 270-279.

[20] B. Dubois, H.H. Feldman, C. Jacova, H. Hampel, J.L. Molinuevo, K. Blennow, et al, Advancing research diagnostic criteria for Alzheimer's disease: the IWG-2 criteria, Lancet Neurol. 13 (2014) 614-629.

[21] American Psychiatric Association, Diagnostic and Statistical Manual of Mental Disorders: DSM-5 t.e.W., D.C. (2013).

[22] A. Martorana, G.M. Sancesario, Z. Esposito, M. Nuccetelli, R. Sorge, A. Formosa, et al., Plasmin system of Alzheimer's disease patients: CSF analysis, J. Neural Transm. (Vienna) 119 (2012) 763-769.

[23] T. Schirinzi, G. Di Lazzaro, G.M. Sancesario, V.L. Colona, E. Scaricamazza, N.B. Mercuri, et al., Levels of amyloid-beta-42 and CSF pressure are directly related in patients with Alzheimer's disease, J. Neural Transm. (Vienna) 124 (2017) $1621-1625$.

[24] S. Rossi, C. Motta, V. Studer, F. Barbieri, F. Buttari, A. Bergami, et al., Tumor necrosis factor is elevated in progressive multiple sclerosis and causes excitotoxic neurodegeneration, Mult. Scler. 20 (2014) 304-312.

[25] N. Siller, J. Kuhle, M. Muthuraman, C. Barro, T. Uphaus, S. Groppa, et al., Serum neurofilament light chain is a biomarker of acute and chronic neuronal damage in early multiple sclerosis, Mult. Scler. 25 (2019) 678-686.

[26] J.L. Molinuevo, S. Ayton, R. Batrla, M.M. Bednar, T. Bittner, J. Cummings, et al., Current state of Alzheimer's fluid biomarkers, Acta Neuropathol. 136 (2018) 821-853.

[27] G.M. Sancesario, S. Bernardini, Diagnosis of neurodegenerative dementia: where do we stand, now? Ann Transl Med. 6 (2018) 340.

[28] G.M. Sancesario, S. Toniolo, D. Chiasserini, S.G. Di Santo, J. Zegeer, G. Bernardi, et al., The clinical use of cerebrospinal fluid biomarkers for Alzheimer's disease diagnosis: the Italian Selfie, J. Alzheimers Dis. 55 (2017) 1659-1666.

[29] J. Busciglio, D.H. Gabuzda, P. Matsudaira, B.A. Yankner, Generation of beta-amyloid in the secretory pathway in neuronal and nonneuronal cells, Proc. Natl. Acad. Sci. U. S. A. 90 (1993) 2092-2096.

[30] C.E. Shepherd, S. Bowes, D. Parkinson, M. Cambray-Deakin, R.C. Pearson, Expression of amyloid precursor protein in human astrocytes in vitro: isoformspecific increases following heat shock, Neuroscience. 99 (2000) 317-325.

[31] D. Scheuner, C. Eckman, M. Jensen, X. Song, M. Citron, N. Suzuki, et al., Secreted amyloid beta-protein similar to that in the senile plaques of Alzheimer's disease is increased in vivo by the presenilin 1 and 2 and APP mutations linked to familial Alzheimer's disease, Nat. Med. 2 (1996) 864-870.

[32] J.S. Miners, S. Baig, J. Palmer, L.E. Palmer, P.G. Kehoe, S. Love, Abeta-degrading enzymes in Alzheimer's disease, Brain Pathol. 18 (2008) 240-252.

[33] O. Hansson, H. Zetterberg, E. Vanmechelen, H. Vanderstichele, U. Andreasson, E. Londos, et al., Evaluation of plasma Abeta(40) and Abeta(42) as predictors of conversion to Alzheimer's disease in patients with mild cognitive impairment, Neurobiol. Aging 31 (2010) 357-367.

[34] J. Kuhle, C. Barro, U. Andreasson, T. Derfuss, R. Lindberg, A. Sandelius, et al. Comparison of three analytical platforms for quantification of the neurofilament light chain in blood samples: ELISA, electrochemiluminescence immunoassay and Simoa, Clin. Chem. Lab. Med. 54 (2016) 1655-1661.

[35] S.Y. Yang, M.J. Chiu, T.F. Chen, H.E. Horng, Detection of plasma biomarkers using Immunomagnetic reduction: a promising method for the early diagnosis of Alzheimer's disease, Neurol Ther. 6 (2017) 37-56.

[36] S. Palmqvist, P.S. Insel, H. Zetterberg, K. Blennow, B. Brix, E. Stomrud, et al., Accurate risk estimation of beta-amyloid positivity to identify prodromal Alzheimer's disease: cross-validation study of practical algorithms, Alzheimers Dement. 15 (2019) 194-204.

[37] A. Nakamura, N. Kaneko, V.L. Villemagne, T. Kato, J. Doecke, V. Dore, et al., High performance plasma amyloid-beta biomarkers for Alzheimer's disease, Nature. 554 (2018) 249-254.

[38] G.M. Sancesario, S. Bernardini, Alzheimer's disease in the omics era, Clin. Biochem. 59 (2018) 9-16.

[39] N.J. Ashton, A.J. Nevado-Holgado, I.S. Barber, S. Lynham, V. Gupta, P. Chatterjee, et al., A plasma protein classifier for predicting amyloid burden for preclinical Alzheimer's disease, Sci. Adv. 5 (2019) eaau7220.

[40] N. Kaneko, A. Nakamura, Y. Washimi, T. Kato, T. Sakurai, Y. Arahata, et al., Novel plasma biomarker surrogating cerebral amyloid deposition, Proc Jpn Acad Ser B Phys Biol Sci. 90 (2014) 353-364.

[41] S. Janelidze, E. Stomrud, S. Palmqvist, H. Zetterberg, D. van Westen, A. Jeromin, et al., Plasma beta-amyloid in Alzheimer's disease and vascular disease, Sci. Rep. 6 (2016) 26801.

[42] M. Gisslen, R.W. Price, U. Andreasson, N. Norgren, S. Nilsson, L. Hagberg, et al., Plasma concentration of the Neurofilament light protein (NFL) is a biomarker of CNS injury in HIV infection: a cross-sectional study, EBioMedicine. 3 (2016) $135-140$.

[43] K.Y. Tzen, S.Y. Yang, T.F. Chen, T.W. Cheng, H.E. Horng, H.P. Wen, et al., Plasma Abeta but not tau is related to brain PiB retention in early Alzheimer's disease, ACS Chem. Neurosci, 5 (2014) $830-836$. 
[44] M.J. Chiu, Y.F. Chen, T.F. Chen, S.Y. Yang, F.P. Yang, T.W. Tseng, et al., Plasma tau as a window to the brain-negative associations with brain volume and memory function in mild cognitive impairment and early Alzheimer's disease, Hum. Brain Mapp. 35 (2014) 3132-3142.

[45] M.J. Chiu, S.Y. Yang, H.E. Horng, C.C. Yang, T.F. Chen, J.J. Chieh, et al., Combined plasma biomarkers for diagnosing mild cognition impairment and Alzheimer's disease, ACS Chem. Neurosci. 4 (2013) 1530-1536.

[46] H. Zetterberg, D. Wilson, U. Andreasson, L. Minthon, K. Blennow, J. Randall, et al., Plasma tau levels in Alzheimer's disease, Alzheimers Res. Ther. 5 (2013) 9.

[47] T. Wang, S. Xiao, Y. Liu, Z. Lin, N. Su, X. Li, et al., The efficacy of plasma biomarkers in early diagnosis of Alzheimer's disease, Int J Geriatr Psychiatry. 29 (2014) 713-719.

[48] D.L. Sparks, R.J. Kryscio, M.N. Sabbagh, C. Ziolkowski, Y. Lin, L.M. Sparks, et al., Tau is reduced in AD plasma and validation of employed ELISA methods, Am J Neurodegener Dis. 1 (2012) 99-106.

[49] S. Krishnan, P. Rani, Evaluation of selenium, redox status and their association with plasma amyloid/tau in Alzheimer's disease, Biol. Trace Elem. Res. 158 (2014) $158-165$.

[50] N. Mattsson, H. Zetterberg, S. Janelidze, P.S. Insel, U. Andreasson, E. Stomrud, et al., Plasma tau in Alzheimer disease, Neurology. 87 (2016) 1827-1835.

[51] C.C. Yang, M.J. Chiu, T.F. Chen, H.L. Chang, B.H. Liu, S.Y. Yang, Assay of plasma phosphorylated tau protein (threonine 181) and Total tau protein in early-stage Alzheimer's disease, J. Alzheimers Dis. 61 (2018) 1323-1332.

[52] L.F. Lue, A. Guerra, D.G. Walker, Amyloid Beta and tau as Alzheimer's disease blood biomarkers: promise from new technologies, Neurol Ther. 6 (2017) 25-36.

[53] P. Lewczuk, N. Ermann, U. Andreasson, C. Schultheis, J. Podhorna, P. Spitzer, et al., Plasma neurofilament light as a potential biomarker of neurodegeneration in Alzheimer's disease, Alzheimers Res. Ther. 10 (2018) 71.

[54] J.C. Rojas, A. Karydas, J. Bang, R.M. Tsai, K. Blennow, V. Liman, et al., Plasma neurofilament light chain predicts progression in progressive supranuclear palsy, Ann Clin Transl Neurol. 3 (2016) 216-225.

[55] O. Preische, S.A. Schultz, A. Apel, J. Kuhle, S.A. Kaeser, C. Barro, et al., Serum neurofilament dynamics predicts neurodegeneration and clinical progression in presymptomatic Alzheimer's disease, Nat. Med. 25 (2019) 277-283.

[56] N.J. Ashton, M. Scholl, K. Heurling, E. Gkanatsiou, E. Portelius, K. Hoglund, et al., Update on biomarkers for amyloid pathology in Alzheimer's disease, Biomark. Med 12 (2018) 799-812.

[57] M. Francois, C.F. Bull, M.F. Fenech, W.R. Leifert, Current state of saliva biomarkers for aging and Alzheimer's disease, Curr. Alzheimer Res. 16 (2019) 56-66.

[58] A. Formosa, M.C. Piro, R. Docimo, P. Maturo, D.R. Sollecito, M. Kalimutho, et al., Salivary miRNAome profiling uncovers epithelial and proliferative miRNAs with differential expression across dentition stages, Cell Cycle 10 (2011) 3359-3368.

[59] C. Santone, V. Dinallo, M. Paci, S. D'Ottavio, G. Barbato, S. Bernardini, Saliva metabolomics by NMR for the evaluation of sport performance, J. Pharm. Biomed. Anal. 88 (2014) 441-446.

[60] F. Bermejo-Pareja, D. Antequera, T. Vargas, J.A. Molina, E. Carro, Saliva levels of Abeta1-42 as potential biomarker of Alzheimer's disease: a pilot study, BMC Neurol. 10 (2010) 108.

[61] M. Lee, J.P. Guo, K. Kennedy, E.G. McGeer, P.L. McGeer, A method for diagnosing Alzheimer's disease based on salivary amyloid-beta protein 42 levels, J. Alzheimers Dis. 55 (2017) 1175-1182.

[62] C.B. Kim, Y.Y. Choi, W.K. Song, K.B. Song, Antibody-based magnetic nanoparticle immunoassay for quantification of Alzheimer's disease pathogenic factor, J. Biomed. Opt. 19 (2014) 051205.

[63] H.C. Lau, I.K. Lee, P.W. Ko, H.W. Lee, J.S. Huh, W.J. Cho, et al., Non-invasive screening for Alzheimer's disease by sensing salivary sugar using Drosophila cells expressing gustatory receptor ( $\mathrm{Gr} 5 \mathrm{a}$ ) immobilized on an extended gate ion-sensitive field-effect transistor (EG-ISFET) biosensor, PLoS One 10 (2015) e0117810.

[64] M. Shi, Y.T. Sui, E.R. Peskind, G. Li, H. Hwang, I. Devic, et al., Salivary tau species are potential biomarkers of Alzheimer's disease, J. Alzheimers Dis. 27 (2011) 299-305.

[65] N.J. Ashton, M. Ide, M. Scholl, K. Blennow, S. Lovestone, A. Hye, et al., No association of salivary total tau concentration with Alzheimer's disease, Neurobiol. Aging 70 (2018) 125-127.

[66] G. Kallo, M. Emri, Z. Varga, B. Ujhelyi, J. Tozser, A. Csutak, et al., Changes in the chemical barrier composition of tears in Alzheimer's disease reveal potential tear diagnostic biomarkers, PLoS One 11 (2016) e0158000.

[67] C.D. Orru, M. Bongianni, G. Tonoli, S. Ferrari, A.G. Hughson, B.R. Groveman, et al., A test for Creutzfeldt-Jakob disease using nasal brushings, N. Engl. J. Med. 371 (2014) 519-529.

[68] M. Bongianni, C. Orru, B.R. Groveman, L. Sacchetto, M. Fiorini, G. Tonoli, et al., Diagnosis of human prion disease using real-time quaking-induced conversion testing of olfactory mucosa and cerebrospinal fluid samples, JAMA Neurol. 74 (2017) 155-162.

[69] T. Etgen, M. Chonchol, H. Forstl, D. Sander, Chronic kidney disease and cognitive impairment: a systematic review and meta-analysis, Am. J. Nephrol. 35 (2012) 474-482.

[70] K. Deckers, I. Camerino, M.P. van Boxtel, F.R. Verhey, K. Irving, C. Brayne, et al., Dementia risk in renal dysfunction: a systematic review and meta-analysis of prospective studies, Neurology. 88 (2017) 198-208.

[71] F. Yao, X. Hong, S. Li, Y. Zhang, Q. Zhao, W. Du, et al., Urine-based biomarkers for Alzheimer's disease identified through coupling computational and experimental methods, J. Alzheimers Dis. 65 (2018) 421-431.

[72] S.M. de la Monte, J.R. Wands, Alzheimer-associated neuronal thread protein-induced apoptosis and impaired mitochondrial function in human central nervous system-derived neuronal cells, J. Neuropathol. Exp. Neurol. 60 (2001) 195-207.

[73] S.M. de la Monte, G.J. Chen, E. Rivera, J.R. Wands, Neuronal thread protein regulation and interaction with microtubule-associated proteins in SH-Sy5y neuronal cells, Cell. Mol. Life Sci. 60 (2003) 2679-2691.

[74] P.J. Kahle, M. Jakowec, S.J. Teipel, H. Hampel, G.M. Petzinger, D.A. Di Monte, et al., Combined assessment of tau and neuronal thread protein in Alzheimer's disease CSF, Neurology. 54 (2000) 1498-1504.

[75] N. Zhang, L. Zhang, Y. Li, M.L. Gordon, L. Cai, Y. Wang, et al., Urine AD7c-NTP predicts amyloid deposition and symptom of agitation in patients with Alzheimer's disease and mild cognitive impairment, J. Alzheimers Dis. 60 (2017) 87-95.

[76] S.M. de la Monte, J.R. Wands, The AD7C-NTP neuronal thread protein biomarker for detecting Alzheimer's disease, Front. Biosci. 7 (2002) d989-d996.

[77] J. Zhang, C.H. Zhang, R.J. Li, X.L. Lin, Y.D. Chen, H.Q. Gao, et al., Accuracy of urinary AD7c-NTP for diagnosing Alzheimer's disease: a systematic review and meta-analysis, J. Alzheimers Dis. 40 (2014) 153-159.

[78] G.M. Sancesario, M.T. Cencioni, Z. Esposito, G. Borsellino, M. Nuccetelli, A. Martorana, et al., The load of amyloid-beta oligomers is decreased in the cerebrospinal fluid of Alzheimer's disease patients, J. Alzheimers Dis. 31 (2012) 865-878. 